\title{
A multi-wavelength campaign to study giant pulses from the Crab Pulsar
}

\author{
Walid Majid \\ JPL/Caltech, USA \\ email: majidw@gmail.com
}

\begin{abstract}
We are currently undertaking a monitoring campaign with NASA 70-m antennas to capture a large sample of Crab Giant Pulses (CGP) at multiple radio wavelengths. The goal of this campaign is to carry out a correlation study of CGPs at radio frequencies with pulsed emission from the Crab pulsar with Fermi photons at X-ray. After a year of this study, we expect around 200 Fermi photons to coincide with a CGP radio-frequency detection, allowing us to either confirm a predicted correlation in average gamma-ray pulsed flux increase with GP emission, or place a tight upper limit, at least a factor of 10 more constraining than previous work. We will report on the status of this campaign and will present our preliminary results and prospects for future improvements in receivers and back-end instrumentation.
\end{abstract}

